\title{
APPLICATION OF SURFACE MICROMACHINING TO EMBRYO LABELING
}

\author{
Lin Wang and David J. Beebe \\ University of Illinois at Urbana Champaign \\ Department of Electrical and Computer Engineering \\ and the Beckman Institute \\ Urbana, IL 61801
}

\author{
Allen R. Williams and Kim D. Easley \\ Louisiana Tech University \\ Department of Animal Science \\ Ruston, LA 71272
}

\begin{abstract}
Surface micromachining techniques are employed to develop two novel embryo labeling techniques. Polysilicon micro branding irons are fabricated and used to create $30 \mu \mathrm{m} \times 30 \mu \mathrm{m}$ brands on the zona of bovine embryos. Patterned polysilicon micro tags are fabricated and attached to bovine embryos with agarose gel. Both methods demostrate the potential for batch labeling of biological objects.
\end{abstract}

\section{INTRODUCTION}

Embryo transfer and in vitro fertilization are rapidly growing fields in both animal and human reproduction. Since the first report of in vitro fertilization of in vitro matured bovine follicular oocytes in 1977, the procedures have become widely used [1]. After retrieval from female donors, immature oocytes are matured, fertilized, cultured and finally implanted back into cows. The implanted embryos can worth $\$ 300-\$ 1,500$ each. It is critical that embryos from each donor be correctly identified through all stages of culture and manipulation. The only method currently available for oocyte/embryo identification is manual labeling of embryo containers. This method is subject to logistical error and contributes to low survivability of embryos. Embryos with unique pedigrees must be cultured separately in order to trace their development. The survival rate of the embryos is consequently reduced as embryos cultured in groups have a significantly higher survival-to-hatching rate versus those cultured separately [2]. Therefore it is important to develop a new method for the oocyte/embryo identification capable of labeling individual embryos. Such a method would enhance survival rate and reduce the chance of mislabeling.

\section{METHODOLOGY}

A bovine embryo has a spherical - structure, composed of the inner mass cells (yolk) and zona pellucida (zona). The yolk is concentrically surrounded by the zona. The diameter of the bovine embryo is typically $100 \mu \mathrm{m}$. The zona is a gel-like protein structure, with a thickness of $10-15 \mu \mathrm{m}$.

\section{ELECTROTHERMAL BRANDING}

Electrothermal branding uses electrical energy to increase the temperature of a local region on the embryo surface to the point where molecular destruction or tissue damage occurs. The damaged area, if visible, can be used as a label. The extent of the damage depends on the heat intensity at the iron tip and duration of heat deposition. Control of the heat deposition, particularly in a small well defined region, is an intricate but necessary manipulation to make a label on the region of interest as well as to avoid unnecessary damage to other regions of the embryo. By passing electric current through a miniature patterned polysilicon heating element, a micro branding iron can be realized. By touching the embryo on the hot surface of a branding iron, the pattern of the iron can be burned on the zona surface.

\section{TAG-ATTACHMENT LABELING}

Tag-attachment labeling involves adhering a thin (2$4 \mu \mathrm{m}), 10 \mu \mathrm{m} \times 10 \mu \mathrm{m}$ to $50 \mu \mathrm{m} \times 50 \mu \mathrm{m}$ patterned polysilicon tag to the surface of the embryo or implanting the tag into the perivitelline space. The unique patterns on the tags serve as the labels. Individual micro tags are suspended over cavities on a substrate via small connecting bars. By breaking or removing the connecting bars, the tags will be released. The released tags can be picked up using micro pipettes via vacuum or electrostatic attraction. Organic glues, such as agarose gel, can be used to adhere the tags to the embryo surfaces.

\section{FABRICATION}

The micro branding irons and tags were fabricated via the three-layer polysilicon surface micromachining process of the Multi-User MEMS Process (MUMPs) program at the MEMS Technology Applications Center in North Carolina. Referring to Figure 1, a 500nm thick low stress silicon nitride layer was first deposited on a phosphorous doped n-type (100) silicon wafer as the electrical isolation layer. Next, a $2.0 \mu \mathrm{m}$ PSG (phosphosilicate glass) oxide, a $2.0 \mu \mathrm{m}$ polysilicon, $1.5 \mu \mathrm{m}$ polysilicon, and a 150nm PSG were deposited in sequence as the sacrificial, structure and etch mask layers, respectively. The wafer was then lithographically pattemed and RIE etched. Finally, a $0.5 \mu \mathrm{m}$ metal layer was deposited to form the leads for the branding iron and the release mask for the tags. To release the tags a $48 \%$ potassium hydroxide $(\mathrm{KOH})$ etchant was used at $85^{\circ} \mathrm{C}$ for $=30$ minutes. The gold lead material and the PSG layer served as top and bottom masks for the tags. The connecting bars were etched via undercutting. Finally, the tags/irons were released/suspended by removal of the PSG sacrificial layer.

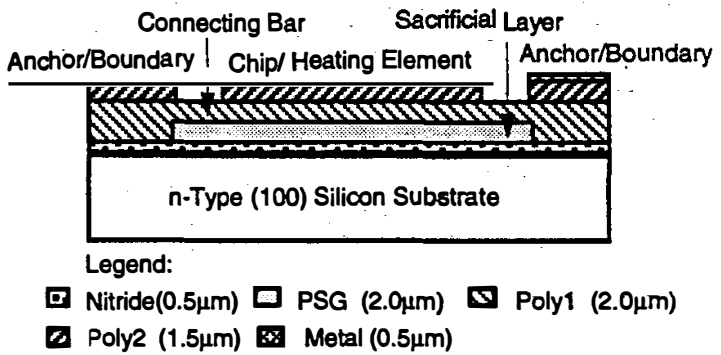

Figure 1 Side view of micro branding iron or micro tag 


\section{RESULTS AND DISCUSSION}

\section{ELECTROTHERMAL BRANDING}

Hundreds of $30 \mu \mathrm{m} \times 30 \mu \mathrm{m} \times 3.5 \mu \mathrm{m}$ polysilicon branding irons with $\mathbf{2 7}$ different shapes were designed and fabricated on a $1 \mathrm{~cm} \times \mathrm{lcm}$ silicon die. Figure 2(a) shows a typical micro branding iron array. The irons are connected to the anchor leads and suspended over the substrate. Referring to Figure 2(b), an embryo was held on the tip of a micropipette via vacuum and then touched to the hot surface of a $32 \mu \mathrm{m} \times 32 \mu \mathrm{m} \times 3.5 \mu \mathrm{m}$ serpentine branding iron. Figure 3(a) shows the result. Three bum marks were formed in a region of $\approx 30 \mu \mathrm{m} \times 30 \mu \mathrm{m}$ on the zona surface. The linewidth of the brand is $=5 \mu \mathrm{m}$. The discontinuity of the marks may be a result of the nonplanar embryo surface causing incomplete contact of the iron with the embryo. In addition, there exists a temperature gradient between the positive and negative ends of the iron. The iron region close to the negative lead is cooler and no burn marks formed. Future modifications to the branding iron geometry will allow for a more uniform temperature on the iron surface.

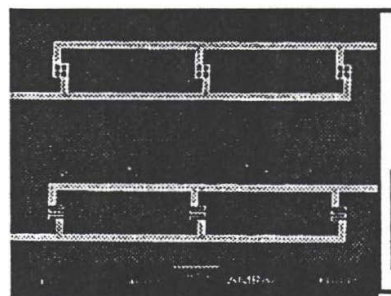

(a)

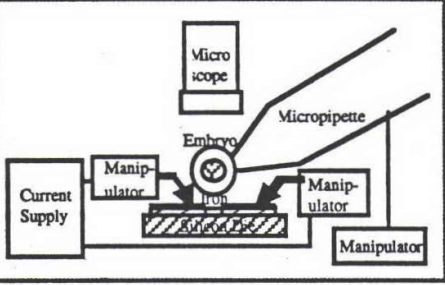

(b)
Figure 2 (a) SEM of a $2 \times 3$ microfabricated array of branding irons (scale bar is $100 \mu \mathrm{m}$ ). (b) Experimental setup for polysilicon iron branding (not to scale).

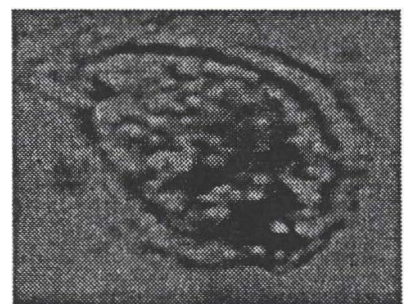

(a)

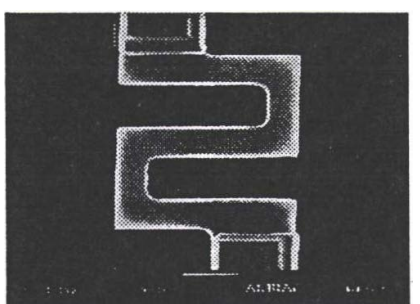

(b)
Figure 3 (a)Three burn marks were created in the region of $\approx 30 \mu \mathrm{m} \times 30 \mu \mathrm{m}$ on the embryo. The brand corresponds to the branding iron shape. (b) The $32 \mu \mathrm{m} \times 32 \mu \mathrm{m} \times 3.5 \mu \mathrm{m}$ polysilicon iron used for the branding (scale bar is $10 \mu \mathrm{m}$ )

\section{TAG-ATTACHMENT LABELING}

Approximately 100,000 polysilicon micro tags with ten unique pattems were fabricated on a $1 \mathrm{~cm} \times 1 \mathrm{~cm}$ silicon die. Figure 4 (b) shows the $30 \mu \mathrm{m} \times 30 \mu \mathrm{m} \times 3.5 \mu \mathrm{m}$ micro tags which are suspended by two or four connecting $2 \mu \mathrm{m} \times 2 \mu \mathrm{m} \times 1.5 \mu \mathrm{m}$ bars. After the connecting bars were removed via $\mathrm{KOH}$ etching, the tags were picked up and held by a micropipette. The embryos were dipped into agarose gel and placed on a glass slide. By touching the micropipette to the embryos, the tags were released and adhered to the gel coating of the embryo. Figure 4 (a) shows the embryo with "E", and "C" micro tags attached.

Compared with the thermal branding, the tag attachment method is more favorable. However, the security of tag adhesion, compatibility, and viability need to be investigated through the entire embryo culture process.

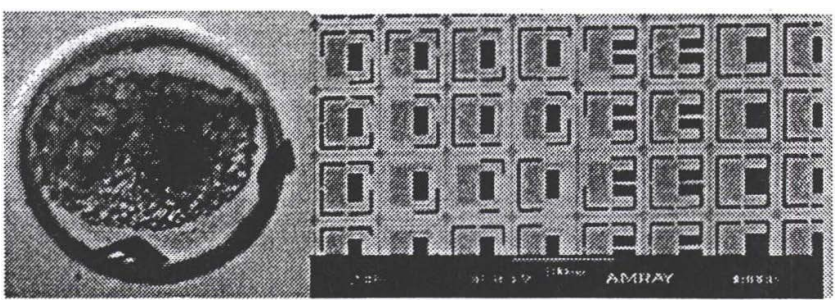

(a)

(b)

Figure 4 (a) Bovine embryos with attached "E," "C" polysilicon tags (diameter of embryo is $100 \mu \mathrm{m}$ ). (b) SEM of microfabricated tags (scale bar is $60 \mu \mathrm{m}$ )

\section{CONCLUSION}

The results presented demonstrate that the electrothermal branding and tag-attachment techniques are potentially feasible for embryo labeling. The use of microfabrication techniques is important and has facilitated the development and manufacturing of the micro branding irons and micro tags. The precise control in geometries, sizes, and patterns of the microfabricated polysilicon branding irons and tags allow the labels to be clearly identified. The high consistency and regular micro scale arrangement of the polysilicon irons and micro tags allows the development of batch labeling techniques. Batch labeling systems are being developed to extend the embryo labeling techniques towards practical use. Biocompatibility and embryo viability studies are currently in progress.

\section{ACKNOWLEDGMENTS}

This project is funded by the Unite States Department of Agriculture (Grant 95-37208-2371). The authors would like to thank the Department of Biomedical Engineering at Louisiana Tech University.

\section{REFERENCE}

1. A. Iritani and K. Niwa, "Capacitation of bull spermatozoma and fertilization in vitro of cattle follicular oocytes matured in culture," J. Reprod. Fertilization, 50: 119-121, (1977).

2. Looney, C. R., B. R. Lindsey, C. L. Gonseth and D. L. Johnson, Commercial aspects of oocyte retrieval and in vitro fertilization (IVF) for embryo production in problem cows, Theriogenology, 41: 67-72, (1994). 